\title{
Nail clipping in onychomycosis and comparison with normal nails and ungual psoriasis*
}

\author{
Flávia Trevisan' ${ }^{1}$, Betina Werner², Rosangela Lameira Pinheiro ${ }^{3}$
}

DOI: http:/ /dx.doi.org/10.1590/abd1806-4841.20198301

\begin{abstract}
BACKGROUND: Of all nail disorders seen in dermatology offices, half of them are due to onychomycosis. The main differential diagnosis is nail psoriasis. The objective of this study was to compare the microscopic findings, other than the presence of fungi, in the clipping of onychomycosis versus normal nails and nail psoriasis.

Methods: Cross-sectional study of onychomycosis cases, analyzed by clipping and compared with data on normal nails and those with nail psoriasis.

RESULTS: Sixty-two onychomycosis samples were compared with 30 normal nails and 50 nails with psoriasis. In onychomycosis, measurement of subungual region, serous lakes, neutrophils and number of layers of parakeratosis are more intense than in psoriasis. Onychocariosis is less common in psoriasis, while bacteria are more frequent. The nail transition zone is more commonly blurred and irregular in onychomycosis.

Conclusion: Clipping helps in the differential diagnosis of onychomycosis and nail psoriasis and may be useful even when fungi are not found.
\end{abstract}

Keywords: Mycology; Nail diseases; Onychomycosis; Pathology; Psoriasis

\section{INTRODUCTION}

Onychomycosis corresponds to $50 \%$ of nail disorders, and the main differential diagnosis is nail psoriasis. The correct diagnosis is essential, and clipping stained with Periodic Acid-Schiff (PAS) is very useful in these investigations. ${ }^{1-5}$

The objective of this study was to compare clippings of onychomycosis with published data on normal nails and ungual psoriasis (UPso). ${ }^{6,7}$

\section{METHODS}

A cross-sectional study was conducted on cases of onychomycosis analyzed using clippings collected between September 2014 and February 2015. These cases were compared with data from two previous publications on normal nails and UPso, performed under the same collection methodologies, processing and terminologies, and evaluated by the same dermatopathologist. ${ }^{6,7}$

The study included patients with distolateral or total dystrophic onychomycosis confirmed by direct examination, culture or clipping. Cases with insufficient subungual material were excluded.

The clipping was performed, using a common nail clipper, by cutting a fragment of $2 \times 5 \mathrm{~mm}$ from the free edge of the most affected nail. The nail clipper was washed and submitted to antisepsis with $70^{\circ}$ alcohol between each case. The clipped material was transported in a plastic, hermetically sealed package, measuring $3 \times 5 \mathrm{~cm}$, and was stained with PAS with digestion by the enzyme diastase after processing. ${ }^{5,6}$

\footnotetext{
Received on 07 June 2016.

Accepted on 04 June 2017.

* Work conducted at the Hospital de Clínicas da Universidade Federal do Paraná, Curitiba (PR), Brazil.

Financial support: None.

Conflict of interest: None.

Department of Dermatology, Hospital de Clínicas, Universidade Federal do Paraná, Curitiba (PR), Brazil.

Department of Pathology, Hospital de Clínicas, Universidade Federal do Paraná, Curitiba (PR), Brazil.

Department of Microbiology and Mycology, Diagnostic Support Unit, Hospital de Clínicas, Universidade Federal do Paraná, Curitiba (PR), Brazil.
}

\section{MAILING AdDREss:}

Flávia Trevisan

E-mail: draflaviatrevisan@ufpr.br 
The microscopic analysis of the plaque assessed thickness in millimeters between the nail surface and the highest point of the nail transition zone (NTZ), with a line perpendicular to the main axis of the piece, clefts, onychocariosis (onychocytes with visible nuclei), and nail granular parakeratosis (NGP; onychocytes within granules in the cytoplasm). The NTZ was described by its definition (blurred or sharp) and by its linearity (regular or irregular). In the subungual region was evaluated for thickness ( $\mathrm{mm}$, from the highest point of NTZ to the last layer of cells visible on the sample), serous lakes, neutrophils, bacteria, and number of layers of subungual parakeratosis (LSP) (Figure 1).

The sample size was calculated using a prevalence of $13.8 \% .{ }^{2}$ Statistical analysis was performed in the EpiInfo ${ }^{\mathrm{TM}}$ software, and descriptive and distribution analyses (non-parametric tests of Chi-square and Mann-Whitney) were performed, with significance level of $\leq 5 \%$.

The study was approved by the committee on bioethics in human research (CAAE 36620114.7.0000.0096). All patients signed the free and informed consent form.

\section{RESULTS}

Sixty-two samples with a diagnosis of onychomycosis were collected. Including the samples from the other studies, total $n$ was 142 .

Of the onychomycosis samples, $62.20 \%$ had a positive direct mycological examination, and $35.48 \%$ had positive cultures, with $68.17 \%$ showing development of T. rubrum. All cases had fungal structures in clippings.

In the 62 cases of onychomycosis, the mean plate thickness was $0.5867 \mathrm{~mm}(0.3900$ to $1.3000 \mathrm{~mm}, \mathrm{SD} \pm 0.2641)$ (Figure 1$)$. Clefts were seen in $61.29 \%$ of the samples, onychocariosis in $51.61 \%$, and NPG in $40.32 \%$ (Figure 2). The average thickness of the subungual region was $0.5361 \mathrm{~mm}(0.2000$ to $1.200 \mathrm{~mm}, \mathrm{SD} \pm 0.3069)$. Serous lakes were present in $64.81 \%$ of samples, neutrophils in $40.74 \%$, and coccus-type bacteria in $33.33 \%$. The mean number of LSP was 10.79

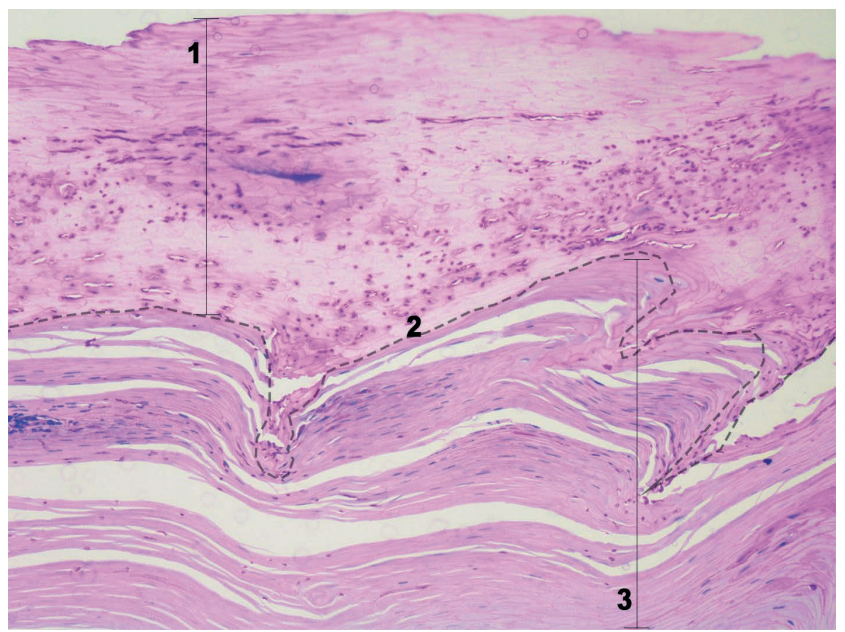

Figure 1: Divisions of the nail. 1- Plate. 2- Nail transition zone (NTZ), demarcated with the dotted line. Here, blurred definition is seen in the right of the image. Linearity is irregular. 3- Subungual region. PAS + fungal structures can be seen on the plaque and the subungual region (Periodic Acid-Schiff, X20)
( 0.0000 to $44.0000, S D \pm 11.7920$ ) (Figure 3 ). In $76.32 \%$ of the samples, the NTZ was blurred (Figure 1), and regarding linearity, $94.74 \%$ were defined as irregular (Figure 3-D).

In the study of normal nails, thirty samples were evaluated. ${ }^{6}$ In the nail psoriasis study, fifty nails with clinical alterations were evaluated, with onycholysis and pitting being the most frequent, followed by leukonychia, oil spot and subungual keratosis ${ }^{7}$ (Table 1). The comparative analysis of the studies is shown in table 2.

\section{DISCUSSION}

Clipping can result in an immediate diagnosis of onychomycosis when fungal structures such as hyphae, pseudohyphae or yeast are seen, and it is a valid complementary test in the case of dermatophytes in particular. ${ }^{8}$ However, other microscopic changes may aid in the investigation of less definitive cases.

It was found that the nail plate tended to be much thicker in onychomycosis than in healthy nails, and although the mean was higher in onychomycosis, there was no statistical difference with UPso cases.

The presence of onychocariosis was more frequent in onychomycosis than in UPso, but there was little difference between onychomycosis and normal nails. Therefore, we suggest that rele-

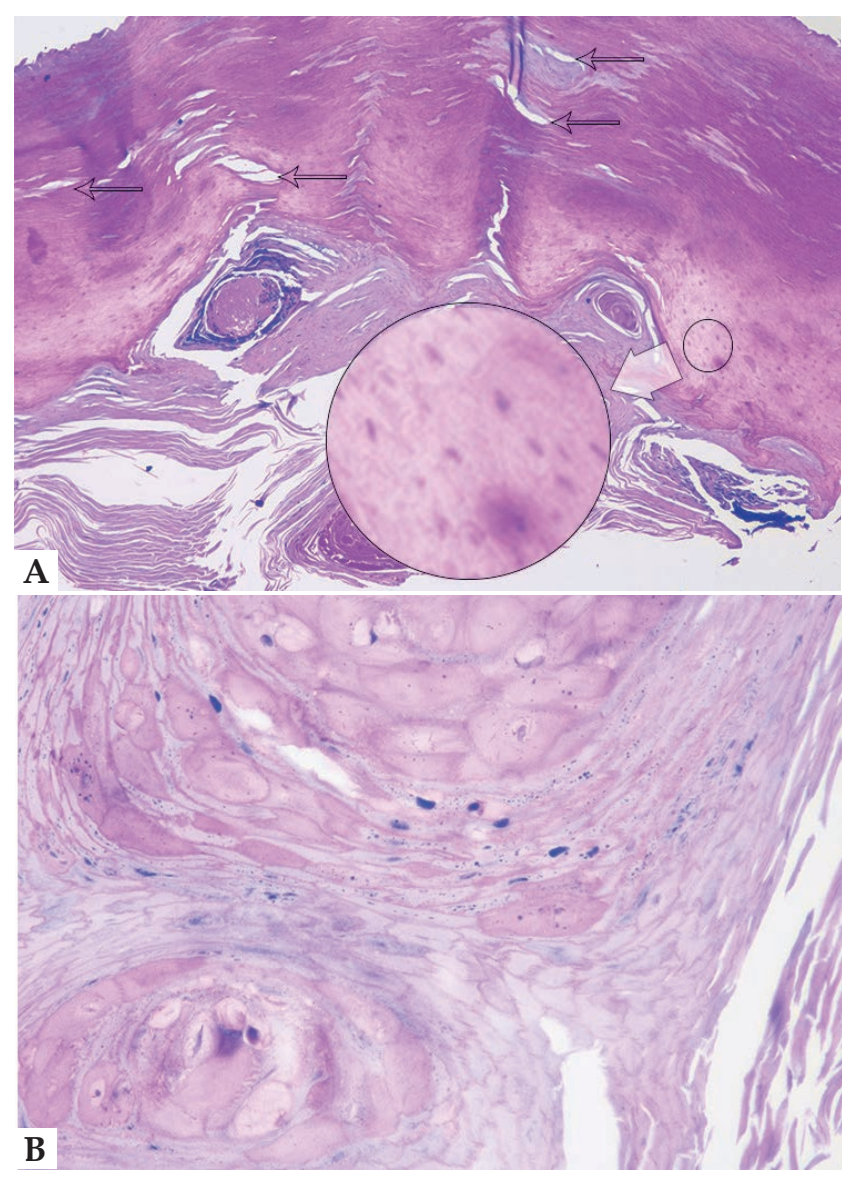

Figure 2: Nail plate with onychomycosis by T. rubrum. A - Clefts (arrows) and onychocariosis (circle in detail) (Periodic Acid-Schiff, X10). B - Nail granular parakeratosis (Periodic Acid-Schiff, X40) 
vance be given to a diminished presence of onychocariosis, as its low frequency is related to UPso cases.

The presence of clefts and NPG cannot be stated as characteristic or exclusive findings of onychomycosis and were therefore cited only for their usefulness in future research.

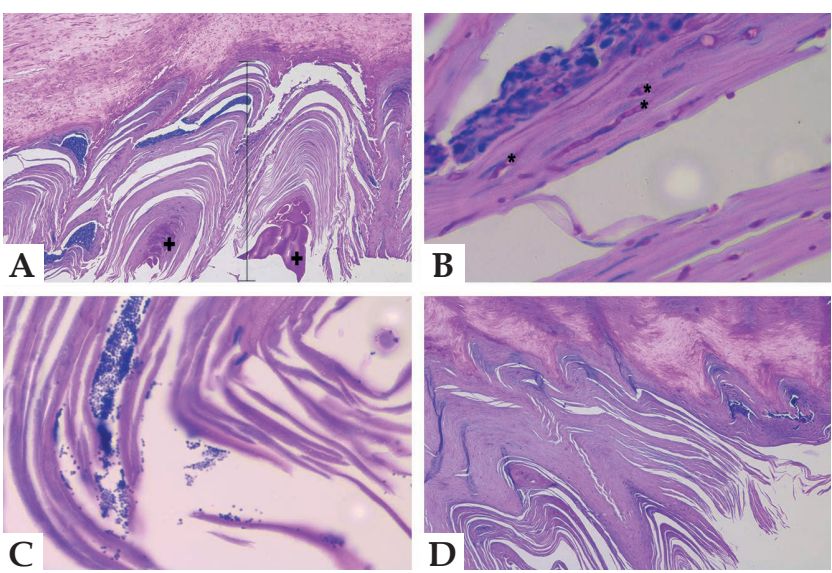

FIgURE 3: Subungual region on nails with onychomycosis by T. rubrum. A - Thickness of the subungual region. Two serous lakes are visible in this image (+) (Periodic Acid-Schiff, X10). B - Neutrophil grouping with hyphae in the vicinity (*) (Periodic Acid-Schiff, X100). C - Bacteria (Periodic Acid-Schiff, X100). D - Marked subungual parakeratosis. The layers of cells stacked in the subungual region and a very irregular NTZ are visible (Periodic Acid-Schiff, X40).
Both the subungual region and the number of LSP are larger in onychomycosis than in UPso, and even larger in comparison to normal nails. The significant difference suggests that the greater the thickness, the greater the suspicion of onychomycosis. It should be noted that extensive and compact parakeratosis is described in psoriasis. ${ }^{9,10}$

Serous lakes and neutrophils are absent in healthy nails, but present in cases of UPso and much more frequent in onychomycosis. These two criteria are very relevant in assessing the degree of inflammation triggered. Future research should consider the relationship between their presence and the severity of the clinical picture, a point that was not evaluated in this study.

The presence of bacteria is more frequent in healthy nails, and much more frequent in cases of UPso, than in nails with onychomycosis. A suggested hypothesis is that competition for the substrates occurs in the presence of fungi, making the nail an environment less favorable to bacteria.

The NTZ can be explained as equivalent to the dermo-epidermal junction, and the linearity as equivalent to the dermal papillae. In this study, the majority of cases presented blurred and irregular NTZ. In cases of UPso, the shape was described as regular and symmetrically and steadily arched. ${ }^{7,10}$

The main limitation of this study is that the data on normal nails and UPso were collected from previous publications and not from control groups designed exclusively for this purpose.

TABLE 1: Results of the findings of onychomycosis and data collected in the studies of normal nails and nail psoriasis

\begin{tabular}{llll} 
Criteria & Onychomycosis & Normal nails (4) & Nail psoriasis (7) \\
\hline Number of samples & 62 & 30 & 50 \\
Plate thickness (mean in mm) & $0.59(0.39-1.3, \mathrm{SD} \pm 0.26)$ & $0.36(0.25-0.50, \mathrm{SD} \pm 0.06)$ & $0.44(0.26-0.85, \mathrm{SD} \pm 3.50)$ \\
Onychocariosis (\%) & 53.33 & 41.00 & 7.00 \\
Subungual thickness (mean in mm) & $0.54(0.2-1.2, \mathrm{SD} \pm 0.09)$ & $0.11(0-0.31, \mathrm{SD} \pm 0.07)$ & $0.19(0-0.7, \mathrm{SD} \pm 0.15)$ \\
Serous lakes (\%) & 64.81 & 0.00 & 46.00 \\
Neutrophils (\%) & 40.74 & 0.00 & 12.00 \\
Layers of subungual parakeratosis (mean) & $10.8(0-44, \mathrm{SD} \pm 11.8)$ & $5.6(1-13, \mathrm{SD} \pm 3.5)$ & $6.9(0-36, \mathrm{SD} \pm 8.12)$ \\
Bacteria (\%) & 32.73 & 60.00 & 74.00 \\
\hline
\end{tabular}

SD: Standard deviation

TABLE 2: Comparative analysis of data from onychomycosis samples and those collected from the literature on normal nails and nail psoriasis

\begin{tabular}{|c|c|c|c|c|c|c|}
\hline \multirow[t]{2}{*}{ Criteria in comparison } & \multicolumn{3}{|c|}{ Onychomycosis X Normal nails } & \multicolumn{3}{|c|}{ Onychomycosis X Nail psoriasis } \\
\hline & Diference & CI 95\% & $\mathrm{p}$ & Diference & CI 95\% & $\mathrm{p}$ \\
\hline Plate thickness (mm) & $0.23(\mathrm{SD} \pm 0.05)$ & $0.33-0.13$ & $<0.0001$ & $0.15(\mathrm{SD} \pm 0.45)$ & $-0.73-1.03$ & 0.7370 \\
\hline Onychocariosis (\%) & $12.33 \%$ & $-9.13-31.91$ & 0.2701 & $46.33 \%$ & $30.17-58.98$ & $<0.0001$ \\
\hline Subungual thickness (mm) & $0.43(\mathrm{SD} \pm 0.02)$ & $0.39-0.47$ & $<0.0001$ & $0.35(\mathrm{SD} \pm 0.02)$ & $0.30-0.40$ & $<0.0001$ \\
\hline Serous lakes (\%) & $64.81 \%$ & $47.98-75.51$ & $<0.0001$ & $18.81 \%$ & $0.38-35.67$ & 0.0469 \\
\hline Neutrophils (\%) & $40.74 \%$ & $24.69-53.16$ & $<0.0001$ & $28.74 \%$ & $12.37-42.71$ & 0.0008 \\
\hline Layers of subungual parakeratosis & $5.2(\mathrm{SD} \pm 2.21)$ & $0.82-9.58$ & 0.0205 & $3.90(\mathrm{SD} \pm 1.96)$ & $0.01-7.79$ & 0.0494 \\
\hline Bacteria $(\%)$ & (-) $27.27 \%$ & $5.68-45.84$ & 0.0134 & (-) $41.27 \%$ & $22.91-55.77$ & $<0.0001$ \\
\hline
\end{tabular}

CI: Confidence interval. SD: Standard deviation. 


\section{CONCLUSION}

The diagnosis of onychomycosis goes beyond the research of fungi in clipping; it may also aid in differential diagnosis with UPso. In onychomycosis, the thickness of the subungual region, the number of subungual parakeratosis layers and the presence of clefts, serous lakes and neutrophils were higher and with statistical significance. In UPso, onychocariosis is less common, and bacteria are more frequent. The NTZ in onychomycosis is predominantly blurred and irregular. $\square$

\section{REFERENCES}

1. Gupta AK, Versteeg SG, Shear NH. Onychomycosis in the 21st century: an update on diagnosis, epidemiology, and treatment. J Cutan Med Surg. 2017;21:525-39.

2. Ghannoum MA, Hajjeh RA, Scher R, Konnikov N, Gupta AK, Summerbell R, et al A large-scale North-American study of fungal isolates from nails: the frequency of onychomycosis, fungal distribution, and antifungal susceptibility patterns. J Am Acad Dermatol. 2000;43:641-8.

3. Bertanha L, Chiacchio ND. Nail clipping in onychomycosis. An Bras Dermatol. 2016;91:688-90.

4. Stephen S, Tosti A, Rubin Al. Diagnostic applications of nail clippings. Dermatol Clin. 2015;33:289-301.

5. Fillus Neto J, Tchornobay AM. How the Nail Clipping helps the dermatologist. An Bras Dermatol. 2009;84:173-6.

6. Werner B, Antunes A. Microscopic examination of normal nail clippings. Dermatol Pract Concept. 2013;3:9-14
7. Werner B, Fonseca GP, Seidel G. Microscopic nail clipping findings in patients with psoriasis. Am J Dermatopathol. 2015;37:429-39.

8. Lavorato FG, Guimarães DA, Premazzi MG, Piñeiro-Maceira JM, BernardesEngemann AR, Orofino-Costa R. Performance of mycology and histopathology tests for the diagnosis of toenail onychomycosis due to filamentous fungi: dermatophyte and non-dermatophyte moulds. Mycoses. 2017;60:587-93.

9. Garbers LE, Slongo H, Fabricio LH, Schmitt JV, Bonalumi A Filho. Incidence, clinical manifestations and clipping of nail psoriasis in the dermatology center of the Hospital Universitário Evangélico de Curitiba. An Bras Dermatol. 2016;91:300-

10. Uber M, Carvalho V0, Abagge KT, Robl Imoto R, Werner B. Clinical features and nail clippings in 52 children with psoriasis. Pediatr Dermatol. 2018;35:202-7.

\section{AUTHORS'CONTRIBUTIONS}

Statistical analysis, Approval of the final version of the manuscript, Conception and planning of the study, Elaboration and writing of the manuscript, Obtaining, analyzing and interpreting the data, Effective participation in research orientation, Intellectual participation in propaedeutic and/or therapeutic conduct of the cases studied, Critical review of the literature, Critical review of the manuscript
Betina Werner
(iD) ORCID 0000-0002-9671-5603

Approval of the final version of the manuscript, Conception and planning of the study, Effective participation in research orientation

Rosangela Lameira Pinheiro 0000-0002-2739-7330

Conception and planning of the study, Intellectual participation in propaedeutic and/or therapeutic conduct of the cases studied

How to cite this article: Trevisan F, Werner B, Pinheiro RL. Nail clipping in onychomycosis and comparison with normal nails and ungual psoriasis. An Bras Dermatol. 2019;94(3):344-7. 